\title{
Zeugung, Entwicklung, Evolution
}

\section{Neue Perspektiven in der Geschichte der Lebenswissenschaften}

Staffan Müller-Wille

Smith, Justin E. H., Hg., 2006. The Problem of Animal Generation in Early Modern Philosophy. Cambridge: Cambridge University Press (=Cambridge Studies in Philosophy and Biology), xiii+456 S., ISBN-13: 978-0-521-84077-4. (AG)

Laubichler, Manfred D./Maienschein, Jane, Hg., 2007. From Embryology to Evo-Devo. A History of Developmental Biology. Cambridge, Mass.: MIT Press (=Dibner Institute Studies in the History of Science and Technology), vii+599 S., ISBN-13: 978-0-262-12283-2. (EE)

Die beiden Sammelbände hätten zu keinem besseren Zeitpunkt erscheinen können. Seit der Publikation des ersten "Arbeitsentwurfs“ der Humangenomsequenz am 26. Juni 2000 werden in den Lebenswissenschaften allenthalben Stimmen laut, dass die Zeiten des „zentralen Dogmas der Molekularbiologie“ mit seiner linearen, vom Gen zum Protein führenden Kausalität vorbei sind und ein neues Denken in Netzwerken und Systemen auf der Tagesordnung steht. Solche Umschwünge lösen in Naturwissenschaft und Philosophie oft das Bedürfnis aus, die Wissenschaftsgeschichte im Rückblick auf verborgene Traditionen und vergessene Alternativen hin zu befragen. Die Autoren beider Bände machen keinen Hehl daraus, dass es ihnen unter anderem auch darum geht, solchen Bedürfnissen entgegenzukommen (AG: 18, EE: 10). Dies führt bei den meisten Beiträgen zu einer internalistischen Perspektive und gibt manchmal sogar Anlass zu offenkundigen Anachronismen: etwa wenn Saul Fisher über „Parallelen“ zwischen Pierre Gassendis' Spekulationen über die Rolle der elterlichen Einbildungskraft bei der Entstehung des Fötus und der „modernen Beschreibung genetischer Dominanz" nachsinnt (AG: 116), oder wenn Stuart A. Newman den englischen Genetiker William Bateson mit seiner „Vibrationstheorie“ der Vererbung als 
einen „Vorläufer“ aktueller Theorien auf dem Gebiet der evolutionären Entwicklungsbiologie vorstellt (EE: 100). Man sollte sich dennoch davor hüten, die Bände aus falsch verstandenem Professionalismus heraus links liegen zu lassen. Zusammengenommen überspannen sie die letzten vierhundert Jahre Geschichte der Lebenswissenschaften und legen überraschende Entwicklungslinien bloß. Wenn auch nur in wenigen Beiträgen auf die kulturellen und politischen Kontexte eingegangen wird, die diese Linien hervortrieben, so verdienen sie doch umso mehr die volle Aufmerksamkeit der Wissenschaftsgeschichte.

Beginnen wir mit dem von Justin H. Smith herausgegebenen Band The Problem of Animal Generation in Early Modern Philosophy. Wie der Titel bereits andeutet, ist die Perspektive hier im Wesentlichen eine philosophiegeschichtliche. In der knappen, aber aufschlussreichen Einleitung macht der Herausgeber deutlich, warum eine solche Betrachtung sich lohnt: Für Mediziner und Naturphilosophen der frühen Neuzeit war es gerade die Zeugung der Tiere - aus heutiger Sicht alles andere als ein elementares Ereignis -, welche die „Mutter aller kausalen Ereignisse“ war (S. 13). Aristoteles' Theorie der vier Ursachen - deren Rezeption durch William Harvey James G. Lennox im ersten Kapitel nachzeichnet - kam insbesondere in der Erklärung der Entstehung individueller Lebewesen voll zur Geltung. Der Cartesianismus, mit seiner Ablehnung formaler und finaler Ursachen, sah sich in dieser Hinsicht vor seine größte Herausforderung gestellt. Im Band wird die Vielfalt an Theorien erkundet, die im 17. und 18. Jahrhundert zur Erklärung der Zeugung der Tiere vorgelegt wurden, wobei sich die meisten Autoren an das Werk einer Person halten: René Descartes (Vincent Aucante), Pierre Gassendi (Saul Fisher), Walter Charleton (Andreas Blank), Daniel Sennert und Gottfried Wilhelm Leibniz (Richard T. W. Arthur), Anne Conway (Deborah Boyle), Nicolas Malebranche (Andrew Pyle), Pierre Bayle (Dennis Des Chene), Georg Ernst Stahl (Francesco Paolo de Ceglia) und Charles Bonnet (François Duchesneau). Nur zwei Beiträge heben sich von dieser werkfokussierten Herangehensweise ab. Justin E. Smith versucht in seinem eigenen Beitrag nachzuvollziehen, warum die mütterliche Einbildungskraft - aus moderner Sicht ein bizarrer Aberglaube - in frühneuzeitlichen Zeugungstheorien einen systematischen Platz einnahm. Und Karen Detlefsen legt eine erfrischend provokante Analyse der Debatte zwischen Albrecht von Haller und Caspar Friedrich Wolff vor, in der sie Unterschieden im Forschungsstil dieser beiden Gelehrten nachgeht. Vier abschließende Kapitel sind Immanuel Kant und seinen Beziehungen zu Pierre Moreau de Maupertuis (John Zammito), zu Johann Friedrich Blumenbach (Brandon C. Look), zu den „spekulativen Ursprungswissenschaften“ seiner Zeit im Allgemeinen (Catherine Wilson) und zur gegenwärtigen Evolutionstheorie (Michael Ruse) gewidmet.

Was den Band besonders wertvoll macht, ist die große Sorgfalt, mit der die Autoren die Interpretation der untersuchten Texte vornehmen. Keine der 
sonst üblichen „Matroschka"-Karikaturen (S. 194) mechanizistischer Präexistenztheorien ist zu finden. Stattdessen lernt man, dass selbst so hartnäckige Verteidiger der Präexistenz wie Nicolas Malebranche oder Charles Bonnet Aussagen treffen konnten, die überraschend epigenetisch klingen (S. 208, 303-305). Vielleicht noch überraschender ist, dass Caspar Friedrich Wolff, der in der zweiten Hälfte des 18. Jahrhunderts epigenetische Vorstellungen wiederbelebte, ausgerechnet Descartes als Kronzeugen aufrief und damit den wohl prominentesten Verfechter einer rein mechanischen Entwicklungstheorie (S. 254-256). Die oft schematische Darstellung, nach der Präformation und Epigenesis in unauflöslichem Widerspruch zueinander standen und wonach die Frühgeschichte der Biologie als eine Abfolge von Phasen beschrieben werden kann, in der mal die eine, mal die andere Doktrin dominierte, muss man wohl aufgeben. Stattdessen, so scheint es, änderte sich die Bedeutung der beiden Begriffe fundamental im Laufe der frühen Neuzeit. Anfänglich ging es um den Moment und die Art der Zeugung eines individuellen Lebewesens, wobei deutlich theologische Motive mitspielten. Traducianisten vertraten die Ansicht, dass die Zeugung eines neuen Individuums durch den elterlichen (meist väterlichen) Samen vermittelt ist, während Kreationisten von der Notwendigkeit einer göttlichen Intervention ausgingen (S. 148).

Wie Smith in der Einleitung hervorhebt (S. 80f.), änderte sich der Rahmen dieser Debatte, als im 18. Jahrhundert nicht mehr die Erzeugung von Einzelwesen, sondern die Reproduktion der Lebewesen in den Vordergrund rückte. Präformation und Epigenese wurden zu Aspekten ein und desselben Prozesses, und die Frage war nun, ob dieser Prozess immer eine präexistente, organische Struktur voraussetzt oder ob man annehmen kann, dass schon strukturlose Materie mit strukturschaffenden Lebenskräften ausgestattet ist (S. 246f.). Einen weiteren epochenübergreifenden Trend macht Smith für die frühe Neuzeit aus. Er bildet so etwas wie einen roten Faden, der sich mehr oder minder explizit durch alle Beiträge zieht: das Auftauchen und die zunehmende Prominenz von Theorien, die Smith „mikrosubstantialistisch“ nennt, da nach ihnen angenommen wird, dass es „erheblich mehr echte, vollauf wirkliche, besondere, primäre Substanzen oder Individuen gibt, als uns vor Augen liegen, und als sich Aristoteles jemals hätte träumen lassen" (S. 9; meine Übersetzung, wie auch die folgenden Zitate). Unsichtbare Lebenskeime begannen demnach nicht nur die Welt außerhalb des Körpers zu bevölkern, sondern auch den Körper selbst. „Ein Tier ist eine Welt, die von Tieren bevölkert ist", heißt es bei Bonnet (S. 294). Die Idee der Mikrosubstantialität hing eng mit Präexistenztheorien zusammen und bereitete wie das neu verstandene Begriffspaar Präformation und Epigenese ebenfalls den Boden für den verallgemeinerten Reproduktionsbegriff des 19. Jahrhunderts. „Es gab“, hält Smith am Ende seiner Einleitung fest, „keinen plötzlichen, verwirrenden Moment mit Darwin: die neue Sicht der Dinge, die wir Darwin zuschreiben, war Jahrhunderte in Vorbereitung" (S. 18). 
Manfred Laubichlers und Jane Maienscheins From Embryology to EvoDevo macht einen weniger geschlossenen Eindruck. Dies liegt zum einen daran, dass dieser Band Autoren versammelt, die aus verschiedenen Disziplinen, einschließlich Soziologie und Biologie, stammen und sich für die Geschichte der Embryologie der letzten 150 Jahre aus sehr unterschiedlichen Gründen interessieren. Zum anderen liegt es aber auch am Gegenstand selbst. Die Herausgeber betonen gleich zu Beginn, dass es ihnen - obwohl es der Titel vielleicht nahe legen könnte - nicht darum geht, eine „lineare und graduelle Entwicklung" nachzuzeichnen von der vergleichenden Embryologie des ausgehenden 19. Jahrhunderts zu „Evo-Devo“. Unter diesem Kürzel (für „evolutionary developmental biology“) firmiert im angelsächsischen Sprachraum das Forschungsfeld, dessen Vertreter sich gegenwärtig mit dem Zusammenhang von Phylogenese und Ontogenese beschäftigen. Vielmehr sollen die einzelnen Beiträge eine „erste Karte“ von der Vielfalt der Forschungstraditionen darstellen, die im 20. Jahrhundert organismischen Ansätzen verpflichtet blieben (S. 9). Manfred Laubichler geht in einem eher historiographisch angelegten Kapitel sogar noch weiter. Er gesteht offen ein, dass es eigentlich unmöglich ist, zu entscheiden, welche Traditionen zur (Vor-)Geschichte von „Evo-Devo“ gehören und welche nicht (S. 15); dass der „epistemische Raum“, in dem nach dem Verhältnis von Ontogenie und Phylogenie gefragt wird, schon seit Beginn des 20. Jahrhunderts ein durch unterschiedliche Forschungspraktiken und -technologien gebrochener, „fragmentarierter" Raum war (S. 20) und dass auch noch nicht ausgemacht ist, ob die zur Zeit vielbeschworene „neue Synthese“ tatsächlich stattfindet (S. 24).

Wenn unter einem "Raum“ etwas verstanden wird, was eine gewisse Struktur besitzt, handelt es sich dann bei der Vorgeschichte von "Evo-Devo“ vielleicht eher um ein „epistemisches Konglomerat“, dessen Zement nur in der Suche nach Alternativen zu klassischer Genetik, Neodarwinismus und dem zentralen Dogma der Molekularbiologie besteht? Lässt man die Beiträge dieses Bandes Revue passieren, so kann man sich dieses Eindrucks kaum erwehren. Frederick B. Churchill zeigt, dass Ernst Haeckels biogenetisches Grundgesetz, das Entwicklungsstudien der vergangenen 150 Jahre vielleicht so etwas wie einen methodischen Leitfaden hätte liefern können, sich schon bald in ein Spektrum "harter" und „weicher" Interpretationen auffächerte und dass gerade darin seine Produktivität lag. Jane Maienschein erläutert, wie Embryologen von Wilhelm Roux bis John Tylor Bonner Zellen mal als formgenerierende Elemente, mal als bloße Resultate morphogenetischer Prozesse behandelten. Weit ausholend, aber etwas schematisch, lotet Gar Allen die methodologischen Spannungen aus, die das ganze 20. Jahrhundert über zwischen deskriptiv-synthetischen und experimentell-analytischen Ansätzen bestanden. Ein Sachverhalt, den John P. Wourms auf die „intellektuelle Arbeitsteilung " zwischen Universitäten auf der einen und Museen und meeresbiologischen Stationen auf der anderen Seite zurückführt (S. 233). 
In Alan C. Loves Beitrag haben wir es dann mit der Paläontologie zu tun, dem Stiefkind der Biologiegeschichte. Die Frage, wie evolutionäre Neuheiten entstehen, ist der Schlüssel zur Geschichte von „Evo-Devo“, stellt Love fest (S. 268) und widmet sich in seinem Aufsatz den Arbeiten des Funktionsmorphologen D. Dwight Davis und des Wirbeltierpaläntologen William K. Gregory. Auf die Geschichte eines anderen Schlüsselbegriffs geht William C. Wimsatt ein: der Begriff der „evolutionären Bürde“, geprägt von Rupert Riedl und weiterentwickelt vor allem von Wimsatt selbst unter der Bezeichnung "generative entrenchment". Scott F. Gilbert zeichnet die Entwicklung von Zellschicksalskarten nach, von E. G. Conklins und Walther Vogts Pionierarbeiten zu Beginn des Jahrhunderts bis zu den computergenerierten Genexpressionskarten unserer Tage. Elihu M. Gerson setzt den bis in die 1960er Jahre dominierenden Forschungsstil der Genetik und Molekularbiologie - gekennzeichnet durch die Überzeugung, dass sich Arten und Organismen auf standardisierte Teile reduzieren lassen - mit dem „amerikanischen Manufaktursystem“ (S. 443) in Beziehung. Die erneute Relevanz entwicklungsbiologischer Fragestellungen führt Gerson auf die „Faszination mit Ebenen, Netzwerken und Komplexität“ (S. 451) zurück, die seit den 1970er Jahren an Boden gewonnen haben soll. Die letzten drei Kapitel schließlich stammen von prominenten Vertretern aus dem Gebiet der Entwicklungsbiologie: Brian K. Hall wirft einen Blick auf die Wurzeln seiner Disziplin im Tischsystem von Anton Dohrns Stazione Zoologica in Neapel und in der Entwicklung eines Mikrotoms, das automatisch Schnittserien von Embryonen herstellte, durch die Cambridge Scientific Instrument Company (an der Charles Darwins jüngster Sohn Horace beteiligt war). Gerd B. Müller stellt klar, dass „Evo-Devo" von heute sich weniger aus der vergleichenden Embryologie des 19. Jahrhunderts herleitet als aus der verbreiteten Unzufriedenheit mit dem Adaptionismus der 1970er Jahre und eine „mechanistische und kausale Perspektive" auf Entwicklungsphänomene anstrebt. Und schließlich wagt Günter P. Wagner einen Blick in die nächste Zukunft von „Evo-Devo“ und auf die methodologischen Herausforderungen, die dieser jungen Disziplin noch bevorstehen.

Man kann die Heterogenität dieses Bandes positiv deuten, nämlich als Zeichen dafür, ein wie wenig zusammenhängendes und vielfältiges Unternehmen die Biologie des 20. und beginnenden 21. Jahrhunderts eigentlich war (und wie viel für die Geschichte, Philosophie und Soziologie der Biologie noch zu tun bleibt). Es gibt aber zwei Beiträge, die in der vorangehenden Revue ausgespart wurden und die noch eine ganz andere Perspektive eröffnen. Marsha Richmonds brilliante Analyse von Richard Goldschmidts entwicklungsgenetischen Arbeiten aus den Jahren 1909 bis 1927 und ihrer Rezeption im angelsächsischen Sprachraum zeigt, dass es sich bei Goldschmidt keinesfalls um einen verkannten Außenseiter handelte. Es war vielmehr gerade die Tatsache, dass er sich genetischer Methoden bediente, die dafür sorgte, 
dass seine unkonventionelle Theorie der quantitativen Genwirkung großen Eindruck bei Biologen aller Fachrichtungen hinterließ (S. 198). James Griesemer geht sogar noch weiter und reiht die Mendel'sche Genetik kurzerhand in die breite Palette von Methoden ein, mit deren Hilfe seit Ende des 19. Jahrhunderts Entwicklungsvorgänge nachgezeichnet werden. Worauf beide Beiträge hindeuten ist, dass es letztlich doch die Methoden der Genetik und später der Molekularbiologie waren, die im 20. Jahrhundert einen Unterschied machten in Hinsicht darauf, wie jahrhundertealte Fragen nach der Entwicklung und Evolution der Organismen beantwortet wurden.

Staffan Müller-Wille

ESRC Centre for Genomics in Society

University of Exeter

Byrne House, St Germans Road

Exeter, Devon EX4 4PJ

Großbritannien

E-Mail: S.E.W.Mueller-Wille@exeter.ac.uk 